\title{
Soft tissue defects of eyelid and malar region: an experience with the McGregor flap
}

\author{
Pradeoth Korambayil Mukundan', Prashanth Varkey Ambookan', Vinoth Kumar Dilliraj ${ }^{2}$ \\ ${ }^{1}$ Jubilee Institute for Surgery of Hand, Aesthetic and Microsurgery, Jubilee Mission Medical College and Research Institute, \\ Thrissur 680005, Kerala, India. \\ ${ }^{2}$ Department of Plastic Surgery and Burns, Vijaya Hospital, Vadapalani, Chennai 600026, Tamil Nadu, India.
}

Address for correspondence: Dr. Pradeoth Korambayil Mukundan, Jubilee Mission Medical College and Research Institute, Bishop Alapatt Road, Thrissur 680005, Kerala, India. E-mail: pradeoth@gmail.com

\begin{abstract}
Aim: Reconstruction of defects of the eyelids and malar region following trauma may result in considerable distortion of the adjacent tissue. A clinical study was undertaken to demonstrate the ability to utilize a modified McGregor flap for reliable soft tissue coverage. Methods: Nine patients with eyelids and malar soft tissue defects were treated over a period of 12 months from July 2013 to June 2014. In this prospective study, a McGregor flap was used for the closure of defects in 9 patients (7 men and 2 women), aged 20-36 years (mean age: 27 years). Three sessions of hyperbaric oxygen therapy were administered postoperatively, and patients received subsequent follow-up. Results: Six patients presented with malar and lower eyelid defects, 2 patients presented with malar defects, and one patient with upper eyelid, lower eyelid and malar defects following trauma. A McGregor flap was performed in all patients. The preexcision defects varied in size from $3 \mathrm{~cm} \times 2 \mathrm{~cm}$ to $4 \mathrm{~cm} \times 3 \mathrm{~cm}$. No secondary procedures were required in any case. Sutures were removed between 7 and 9 days postoperatively. There were no cases of partial or total flap loss over the course of 10-14 months follow-up. Conclusion: The outcome following use of the McGregor flap procedure was functionally and aesthetically satisfactory in all cases. The McGregor flap is a useful option for the reconstruction of defects following trauma to the upper eyelid, lower eyelid, and malar regions.
\end{abstract}

Key words:

Eyelid defects, hyperbaric oxygen therapy, malar defects, McGregor flap

\section{INTRODUCTION}

Traumatic injuries of the malar region and lateral aspect of the upper and lower eyelids are common following a fall or abrasion. There is a need for a method of soft tissue coverage that can simultaneously address all three defects while preventing both a functional deficit and distortion of the adjacent tissue. When compared to the western

\begin{tabular}{|l|l|}
\hline \multicolumn{2}{|c|}{ Access this article online } \\
\hline Quick Response Code: & Website: \\
\hline Dww.parjournal.net \\
\hline
\end{tabular}

population, the potential for scar formation is profound in Asian patients secondary to their skin pigmentation. The McGregor flap provides satisfactory results when utilized for the reconstruction of such injuries.

\section{METHODS}

The study was conducted at the Jubilee Institute for Surgery of Hand, Aesthetics and Microsurgery, Jubilee Mission Hospital, Thrissur, India. The patients were enrolled in the Plastic and Reconstructive Surgery Department of Jubilee Mission Hospital. Nine patients with eyelids and malar soft tissue defects were treated with the McGregor flap during a period of 12 months from July 2013 to June 2014, followed by 3 sessions of hyperbaric oxygen therapy. The postoperative course and subsequent follow-up were noted. All involved patients agreed to have their facial pictures published and signed the consent form. 


\section{RESULTS}

Six patients presented with malar and lower eyelid defects, 2 patients presented with malar defects, and 1 patient with upper and lower eyelid and malar defects following trauma. The patients' ages ranged from 20 to 36 years (with a mean age of 27 years). Among 9 patients, 7 were males and 2 were females. The defects varied in size from $2 \mathrm{~cm} \times 3 \mathrm{~cm}$ to $3 \mathrm{~cm} \times 4 \mathrm{~cm}$. A McGregor flap was performed in all cases. No secondary procedures were required. Sutures were removed between 7 and 9 days postoperatively. There was no evidence of partial or total flap loss during the follow-up period [Table 1].

\section{Patient with upper and lower eyelid and malar defects}

A 26-year-old male patient presented with upper and lower eyelid and malar defects [Figure 1a]. Wound debridement was performed, and a modified McGregor flap was planned for reconstruction of the soft tissue defect [Figure 1b and c]. A modified McGregor flap was performed [Figure 1d and e], which provided coverage of the defect with the use of a Z-plasty at the lateral aspect of the flap for flap advancement. The patient underwent three sessions of hyperbaric oxygen therapy postoperatively. Regular follow-up was performed, and the flap was noted to remain viable [Figure $1 \mathrm{f}$ and $\mathrm{g}$ ].

\section{Patient with lower eyelid and malar defect}

A 36-year-old woman sustained an injury resulting in soft tissue deficits of the right malar and right lower eyelid [Figure 2a]. Wound debridement was carried out with use of the McGregor flap for coverage [Figure 2b]. The patient received three sessions of postoperative hyperbaric oxygen therapy and the flap healed well [Figure 2c].

\section{Patient with malar defect}

A 21-year-old man presented with a defect of the right malar region following a road traffic accident [Figure 3a]. Wound debridement with McGregor flap coverage was performed [Figure 3b]. Postoperatively, three sessions of hyperbaric oxygen therapy were administered. The flap healed well without complications [Figure 3c].

\section{DISCUSSION}

Soft tissue defects of the eyelids and malar regions following trauma may result in considerable disfiguration, functional derangement, and distortion of the adjacent

Table 1: The patients treated with use of the McGregor flap for soft tissue defects in the upper and lower eyelid and malar regions

\begin{tabular}{|c|c|c|c|c|c|}
\hline Patients & $\begin{array}{l}\text { Age (years)/ } \\
\text { gender }\end{array}$ & Trauma/etiology & Site & Defect area $\left(\mathrm{cm}^{2}\right)$ & Flap pattern \\
\hline 1 & 27/male & Road traffic accident & Right malar and lower eyelid regions & $3 \times 4$ & McGregor flap \\
\hline 2 & 23/male & Road traffic accident & Right malar region & $3 \times 3$ & McGregor flap \\
\hline 3 & 25/male & Road traffic accident & Right malar and lower eyelid regions & $3 \times 4$ & McGregor flap \\
\hline 4 & 36/female & Road traffic accident & Left malar and lower eyelid regions & $3 \times 4$ & McGregor flap \\
\hline 5 & 20/female & Road traffic accident & Right malar and lower eyelid regions & $3 \times 4$ & McGregor flap \\
\hline 7 & $35 /$ male & Road traffic accident & Right malar and lower eyelid regions & $4 \times 3$ & McGregor flap \\
\hline 8 & $21 /$ male & Road traffic accident & Right malar region & $3 \times 3$ & McGregor flap \\
\hline 9 & 26/male & Road traffic accident & Right malar and lower and upper eyelid regions & $\begin{array}{c}3 \times 3 \text { in lower part, } \\
3 \times 2 \text { in upper eyelid }\end{array}$ & $\begin{array}{l}\text { Modified } \\
\text { McGregor flap }\end{array}$ \\
\hline
\end{tabular}

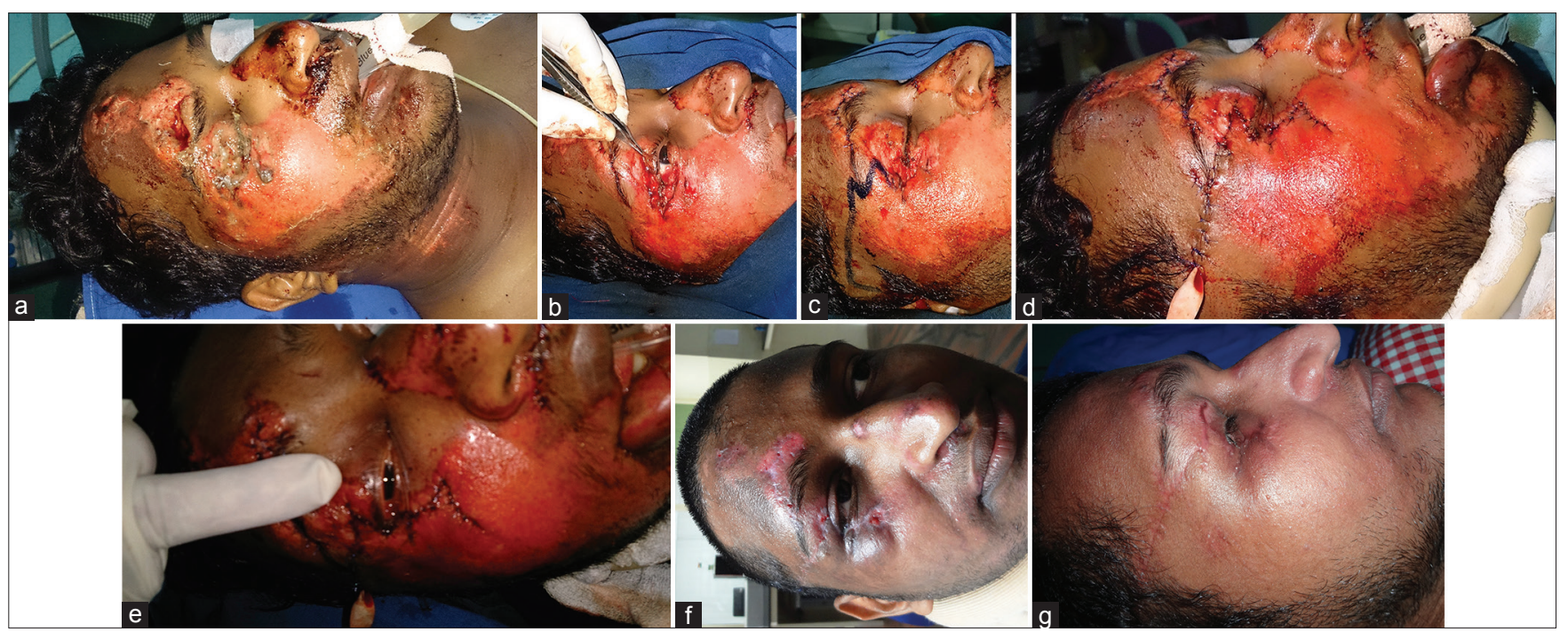

Figure 1: (a) Posttraumatic soft tissue defect, right upper and lower eyelids and malar region; (b) soft tissue defect following surgical debridement; (c) planning of modified McGregor flap; (d) immediate postoperative picture following reconstruction; (e) immediate postoperative anterior view following reconstruction; (f) late postoperative anterior view following reconstruction; $(\mathrm{g})$ late postoperative lateral view following reconstruction 
tissue. Traumatic injuries of the malar region and lateral aspect of the upper and lower eyelids are common following a fall from the motorcycle, or in cases of severe facial abrasion following a fall and drag along a roadway [Figure 4]. There is a need for soft tissue
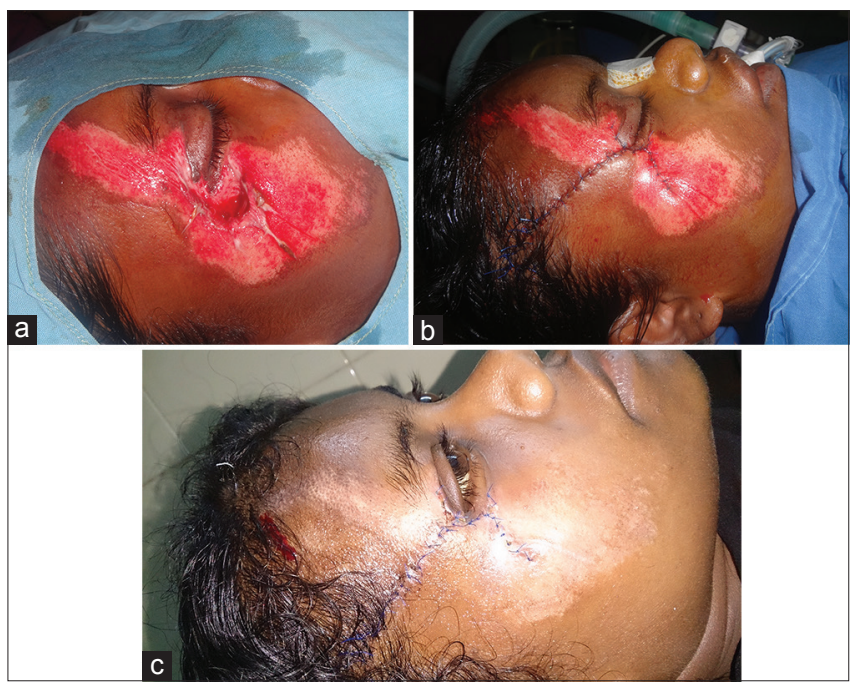

Figure 2: (a) Posttraumatic soft tissue defect, right lower eyelid and malar regions; (b) immediate postoperative picture following reconstruction; (c) late postoperative lateral view following reconstruction

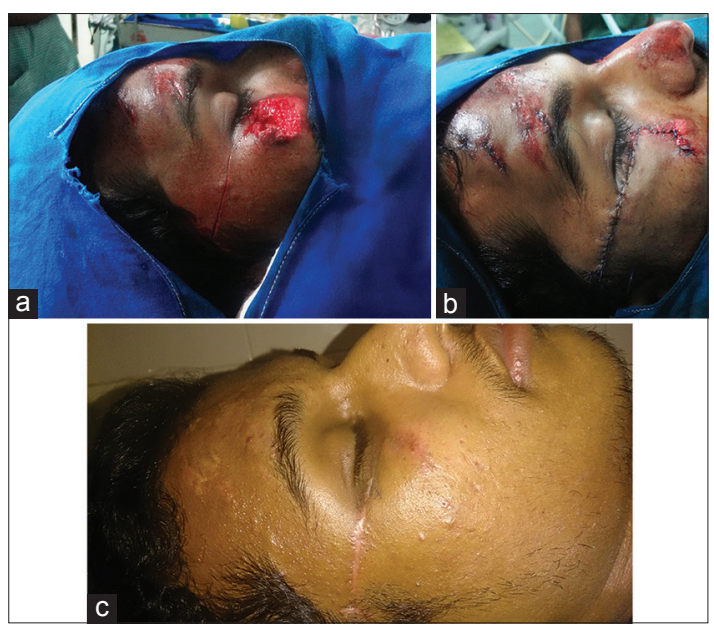

Figure 3: (a) Soft tissue defect, malar region and planning of the McGregor flap; (b) immediate postoperative picture following reconstruction; (c) late postoperative lateral view following reconstruction

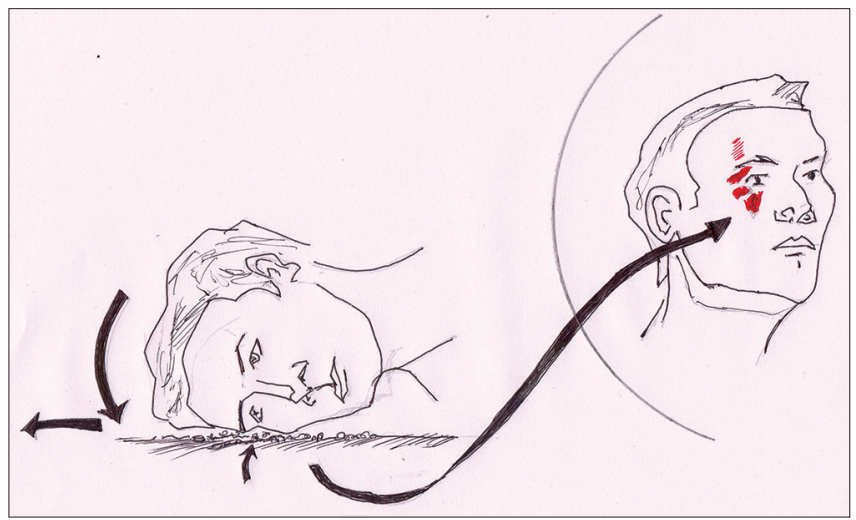

Figure 4: Schematic diagram representing soft tissue defect on the right side of the face following a road traffic accident coverage which addresses all three defects simultaneously while preventing functional deficit and distortion of the adjacent tissue. The ideal reconstruction should avoid creating a "trap door" deformity, dog ear formation, ectropion, and sideburn displacement. ${ }^{[1]}$

Various local flaps such as the rotation flap, transposition flap, advancement flaps, rhomboid flap, bilobed flaps, and "reading man" flaps can be employed for the reconstruction of such defects. ${ }^{[2-6]}$ Most of these flaps have been described for reconstruction of circular defects following tumor resection. Although rhomboid, bilobed and reading man flaps provide soft tissue coverage, they may result in scar formation secondary to the multiple incisions required for flap execution. The Tenzel flap is an advancement-rotation flap in which a semicircular skin-muscle flap is fashioned from the skin lateral to the lateral canthus, and which can be used for both upper and lower eyelids. ${ }^{[7]}$

McGregor devised a flap that adds a Z-plasty to the Mustarde cheek advancement flap for moderate defects of the lower eyelid. ${ }^{17,8]}$ An incision is made lateral to the eyelid, slanted upward gently, and carried into the temporal region. A backcut is then made at the temporal end of the incision and angled medially approximately $30^{\circ}$. A Z-plasty is created, which recruits the vertical laxity from the lateral periorbital region to correct horizontal defects of the lower eyelid. However, the same technique could well be utilized to cover moderate defects in the malar, as well as the upper eyelid region. Similarly by introducing certain modifications [Figure 1c] the same flap could be utilized to reconstruct defects of the eyelids and malar regions.

In our experience, the use of hyperbaric oxygen therapy may result in a favorable outcome in such injuries. Based on our clinical experience, a minimum of three sessions of hyperbaric oxygen therapy contributes to a reduction in edema, which increases the likelihood of flap survival. While steroids may contribute to the anti-edema effect, they may result in associated immunosuppression. ${ }^{[9]}$

The modified McGregor flap is a useful option in the reconstruction of defects of the upper and lower eyelids and malar regions. Hyperbaric oxygen therapy, as an adjuvant to such traumatic facial injuries, will yield better outcomes.

\section{REFERENCES}

I. Mutaf M, Günal E, Temel M. Closure of defects of the malar region.J Craniofac Surg 20I I;22:63I-4

2. Cecchi R, Fancelli L, Troiano M. The "reading man" flap in facial reconstruction: report of I2 cases. Dermatol Online J 20I2; I8: I6.

3. Hayano SM, Whipple KM, Korn BS, Kikkawa DO. Principles of periocular reconstruction following excision of cutaneous malignancy. J Skin Cancer 2012;2012:438502.

4. Saito A, Saito N, Furukawa H, Hayashi T, Oyama A, Funayama E, Minakawa H, Yamamoto Y. Reconstruction of periorbital defects following malignant tumour excision: a report of 50 cases. J Plast Reconstr Aesthet Surg 20 I 2;65:665-70.

5. Yenidunya MO, Demirseren ME, Ceran C. Bilobed flap reconstruction in infraorbital skin defects. Plast Reconstr Surg 2007; I 1 9: 145-50.

6. Mutaf M, Günal E, Temel M. A new technique for closure of infraorbital 
defects. Ann Plast Surg 201 I;67:600-5.

7. Thornton JF, Kenkel JM. Eyelid Reconstruction. Vol. I0. Dallas: Selected Readings in Plastic Surgery, Inc.; 2005. p. 16.

8. Emsen IM. Functional lower lip reconstruction with a modification in McGregor flap technique. Plast Reconstr Surg 2007; I 19:2335-6.

9. Bhutani S, Vishwanath G. Hyperbaric oxygen and wound healing. Indian J Plast Surg 2012;45:316-24.
How to cite this article: Mukundan PK, Ambookan PV, Dilliraj VK. Soft tissue defects of eyelid and malar region: an experience with the McGregor flap. Plast Aesthet Res 2015;2:69-72.

Source of Support: Nil, Conflict of Interest: None declared.

Received: 13-08-2014; Accepted: 04-01-2015 